\title{
Mitomycin C in the Endoscopic Treatment of Laryngotracheal Stenosis: Systematic Review and Proportional Meta-Analysis
}

\author{
Thereza L. O. Queiroga ${ }^{1}$ \\ Antônio J. M. Cataneo ${ }^{2}$ \\ ${ }^{1}$ Department of Otorhinolaryngology and Head and Neck Surgery, \\ Faculdade de Ciências Médicas e Biológicas de Botucatu, \\ Universidade Estadual Paulista, Botucatu, São Paulo, Brazil \\ 2 Department of Surgery, Faculdade de Ciências Médicas e Biológicas de \\ Botucatu, Universidade Estadual Paulista, Botucatu, São Paulo, Brazil
}

\author{
Daniele C. Cataneo ${ }^{2}$ Regina H. G. Martins ${ }^{10}$ Tarcisio A. Reis $^{2}$
}

Int Arch Otorhinolaryngol 2020;24:e112-e124.

\begin{abstract}
Address for correspondence Regina H. G. Martins, MD, PhD, Departamento de Otorrinolaringologia e Cirurgia de Cabeça e Pescoço, Faculdade de Ciências Médicas e Biológicas de Botucatu, Universidade Estadual Paulista, Distrito de Rubião Junior $\mathrm{s} / \mathrm{n}$, 18618-970, Botucatu, SP, Brazil (e-mail: Regina.g.martins@unesp.br).
\end{abstract}

\begin{abstract}
Keywords

- laryngotracheal stenosis

- mitomycin C

- systematic review

- dilation

- endoscopic treatment

Introduction Mitomycin C is a natural antibiotic that has been used to inhibit the proliferation of fibroblasts in scar tissue.

Objective To evaluate the effectiveness and safety of topical Mitomycin C as an adjuvant in the endoscopic treatment of laryngotracheal stenoses.

Data synthesis A systematic review of experimental or observational studies that have evaluated the treatment of laryngotracheal stenoses with the use of topical Mitomycin C was performed. Databases researched: LILACS, PubMed, Embase, Cochrane and Web of Science. Outcomes: resolution (symptom-free time $\geq$ one year), number of procedures required, and complications resulting from the procedure. A total of 15 studies (involving 387 patients) were selected. Mitomycin C was administered to every patient in 11 studies, and in 4 other studies, the patients were separated into 2 groups, 1 receiving mitomycin C, and the other not. The resolution of the stenosis evaluated in 12 studies in which the patients received mitomycin $C$ was of $69 \%$ (95\% confidence interval [95\%Cl]: $61-76 \% ; I^{2}=17.3 \%$ ). A total of $52 \%$ of the patients ( $95 \% \mathrm{Cl}$ : $39-64 \%, 11$ studies; $\mathrm{I}^{2}=64.7 \%$ ) were submitted to a single endoscopic procedure, and $48 \%\left(95 \% \mathrm{Cl}\right.$ : $36-61 \%, 11$ studies; $\left.I^{2}=64.7 \%\right)$ were submitted to more than 1 procedure. Complications (mediastinal and subcutaneous emphysema, dysphonia, laceration or vocal fold paralysis and acute light obstruction) were reported in $9 \%$ of the patients ( $95 \% \mathrm{Cl}$ : $3-18 \%$, 9 studies; $\mathrm{I}^{2}=79.8 \%$ ).

Conclusions The evidence suggests that mitomycin $C$ is an effective and safe option in the endoscopic treatment of laryngotracheal stenosis.
\end{abstract}

\section{Introduction}

The increasing number of patients with postintubation laryngotracheal stenosis has been a challenge because of the lack of an effective treatment modality. The objective of an effective laryngotracheal stenotic treatment should be to ensure adequate airway diameter to allow ventilation and provide symptom relief, which are not achieved in most patients. In many cases, endoscopic treatments and open surgical techniques are used in the same patient; however, when surgical resection is not indicated, endoscopic treatment options are adopted, which have an immediate effect. ${ }^{1}$ received

March 28, 2019

accepted

September 10, 2019
DOI https://doi.org/

10.1055/s-0039-1700582. ISSN 1809-9777.
Copyright (e) 2020 by Thieme Revinter

Publicações Ltda, Rio de Janeiro, Brazil
License terms

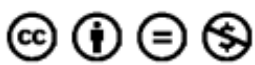


Topical agents have been used as adjuvants in endoscopic treatment, aiming to minimize recurrence rates and prolong the symptom-free period after the intervention. The adjuvant options studied in animal and human models include steroid injections, topical mitomycin C (MMC), topical heparin, 5-fluorouracil, and halofuginone. ${ }^{2}$

Of these options, MMC is highlighted as an antibiotic and antineoplastic agent that inhibits the proliferation of fibroblasts, thereby modulating the healing processes. There is a long history of studies published regarding laryngology research on the effect of MMC on laryngotracheal lesion models in dogs, rabbits, pigs, and rats. The success rate in maintaining the dilation of the stenosis over a long period ranges from $40 \%$ to $70 \%$, with an average of $50 \%{ }^{3}$ Satisfactory results have expanded the search for methods to improve the success rate, ${ }^{3}$ but the overall results of the use of MMC in humans are conflicting.

Although surgical resection of laryngotracheal stenosis with end-to-end anastomosis is effective, conservative options are necessary to treat patients for whom surgery is not indicated. Therefore, the present systematic review was conducted to evaluate the efficacy of MMC as an adjuvant in the endoscopic treatment of laryngotracheal stenosis.

\section{Review of the Literature}

\section{Methodology}

The present systematic review included studies published on the use of topical MMC in the endoscopic treatment of laryngotracheal stenosis. The study design (review of the literature) enabled the exemption of approval from the Research Ethics Committee. Inclusion criteria: experimental or observational studies (with at least 5 participants per group, with more than $80 \%$ of the patients being adolescents or adults, excluding studies that only address children) evaluating endoscopic interventions with adjuvant topical MMC being at least one of the treatment options for laryngotracheal stenosis. Participants: patients with laryngotracheal stenosis. Interventions: endoscopic interventions (mechanical dilation; use of electric scalpel, argon, harmonic, or laser; and cryotherapy) in which MMC was administered to at least one participant group. Outcomes: the primary outcomes include complete or partial resolution of laryngotracheal stenosis, characterized by symptom-free period $\geq 1$ year; the secondary outcomes include the number of procedures with or without the administration of MMC (single or multiple procedures); and complications characterized by the need for immediate intervention or other procedures (acute obstruction, tracheostomy, dysphonia, subcutaneous emphysema, vocal fold laceration, temporary vocal paralysis, among others).

We conducted a search for relevant literature on the following electronic databases: PubMed (from 1966 to November 27, 2018); Embase (from 1973 to November 27, 2018); LILACS (from 1982 to November 27, 2018); Cochrane (from 1993 to November 27, 2018); Web of Science (from 1900 to November 27, 2018); and Clinical Trials (accessed on November 27, 2018). The following search terms were used to find studies on the Medline (PubMed) database: (tracheal stenosis or stenoses, tracheal or stenosis, tracheal or tracheal stenoses) and (bronchoscopy or bronchoscopies or bronchoscopic surgical procedures or bronchoscopic surgical procedure or surgical procedure, bronchoscopic or surgical procedures, bronchoscopic or bronchoscopic surgery or bronchoscopic surgeries or surgeries, bronchoscopic or surgery, bronchoscopic) and (placebos or sham treatment or mitomycin or mitomycin $C$ or mitomycin- $C$ or mitocin-C or mitocin C or mitocinC or NSC-26980 or NSC 26980 or NSC26980 or ametycine or mutamycin or adrenal cortex hormones or hormones, adrenal cortex or corticosteroids or corticoids or lasers or laser or Q-switched lasers or laser, $Q$-switched or lasers, Q-switched or Q-switched lasers or $Q$-switched laser or pulsed lasers or laser, pulsed or lasers, pulsed or pulsed laser or continuous wave lasers or continuous wave laser or laser, continuous wave or lasers, continuous wave or masers or maser or argon or cryotherapy or cryotherapies or therapy, cold or cold therapies or therapies, cold or cold therapy). The same search strategy was applied to each database to achieve greater sensitivity. There was no restriction regarding the language. The reference lists of relevant publications found were analyzed to search for studies not identified with the search strategy.

Selection of studies: two researchers (TLOQ and DCC) independently examined the titles and abstracts to eliminate irrelevant articles, retrieved relevant full texts, identified the location and environment of the studies, the details of the intervention, the date and duration of the studies, and assessed the full texts for compliance with the eligibility criteria. Study authors were contacted, if necessary, to clarify questions related to the study and to decide on its inclusion in this review. Any disagreements were resolved by consensus and discussion with the participation of all of the authors.

Data extraction and management: data from the selected studies were extracted independently by the two reviewers (TLOQ and DCC). A standard form was used to obtain the following information from the extracted studies: type of treatment, total number of participants, number of patients for each intervention, follow-up period, number of interventions performed, evaluated outcomes, type of study, age of the patients and participants, and initial and final classification of laryngotracheal stenosis.

Assessments of the risk of bias were performed for randomized controlled trials (RCTs) by using the "Risk of Bias" tool for Cochrane reviews. These assessments were not performed for observational studies because of the lack of consensus for their application in these studies; however, we consider that they are biased and subject to the effect of confounding factors. The two reviewers independently evaluated each study. Any disagreements were resolved by consensus, or by consultation with the third author (RHGM).

Measures of treatment effect: two measurement forms for the interventional effect were used: a proportional metaanalysis using the StatsDirect (StatsDirect Ltd, Birkenhead, Merseyside, UK) software, version 3.0.121, was performed for the studies that only evaluated the effects of the MMC intervention. ${ }^{4}$ Forest plots were drawn to summarize dichotomous outcome data presented as a proportion with their corresponding 95\% confidence intervals (95\%CIs). The Review Manager 
(RevMan, The Nordic Cochrane Centre, Copenhagen, Denmark) software, version 5.3 was used to analyze studies that compared the effects of interventions with and without MMC. The dichotomous outcomes analyzed were considered with a 95\% $\mathrm{Cl}$. The results were also summarized using a forest plot.

To quantify the inconsistencies of the studies used in the meta-analysis, we conducted the heterogeneity test $\mathrm{I}^{2}=[(\mathrm{Q}-\mathrm{df}) / \mathrm{Q}] \times 100 \%$, in which $\mathrm{Q}$ is the Chi squared, and $\mathrm{df}$ is the degree of freedom. In the presence of heterogeneity between the studies, we used the random effect model of the meta-analysis, leaving the fixed-effect model only for heterogeneities equal to zero. The random effect model was planned to perform subgroup analyses according to the location and severity of the stenosis.

\section{Results}

Search results: an initial search for relevant studies was conducted in October 2015, and another search was conducted on November 27, 2018. With these searches, we identified a total of 723 articles from PubMed, Embase, LILACS, Cochrane, and Web of Science databases, and 1 ongoing study in the Clinical Trials database. ${ }^{18}$ - Fig. 1 is the flowchart of the selection process of all studies.

Included studies: in total, 15 studies $^{1,3,5-17}$ (written in English: 14; written in Portuguese: 1) published from 2001 to 2018 (case series: 12; prospective, randomized: 1; casecontrol: 1 ; prospective cohort: 1$)$ were included in the present review ( - Table 1). Of these studies, 10 were conducted in the United States, one in Portugal, one in the United Kingdom, one in India, and one in Brazil, and the last one was a multicenter (Thailand and Germany) study (-Fig. 1).

Characteristics of the included studies: the selected studies involved a total of 387 patients aged between 2 and 79 years (mean age: 46 years). The gender of the patients was only mentioned in 12 studies (men: 116; women: 199). In the remaining 3 studies (72 patients), the gender was not mentioned. Laryngotracheal stenosis was diagnosed by anamnesis, physical examination, imaging methods, endoscopic examinations and, in some cases, intraoperatively. The classification

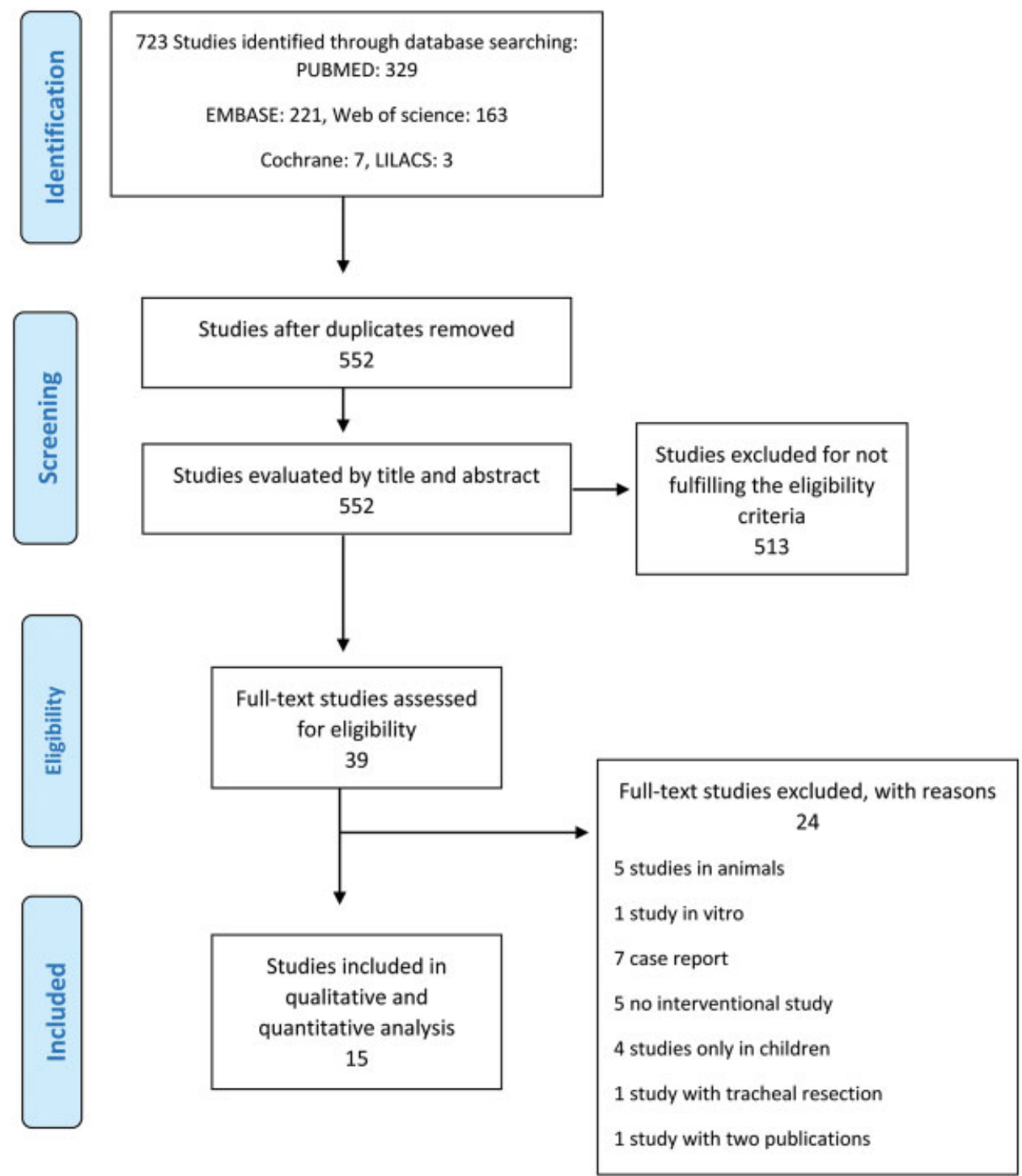

Fig. 1 Flowchart showing the studies identified and evaluated during the review. 


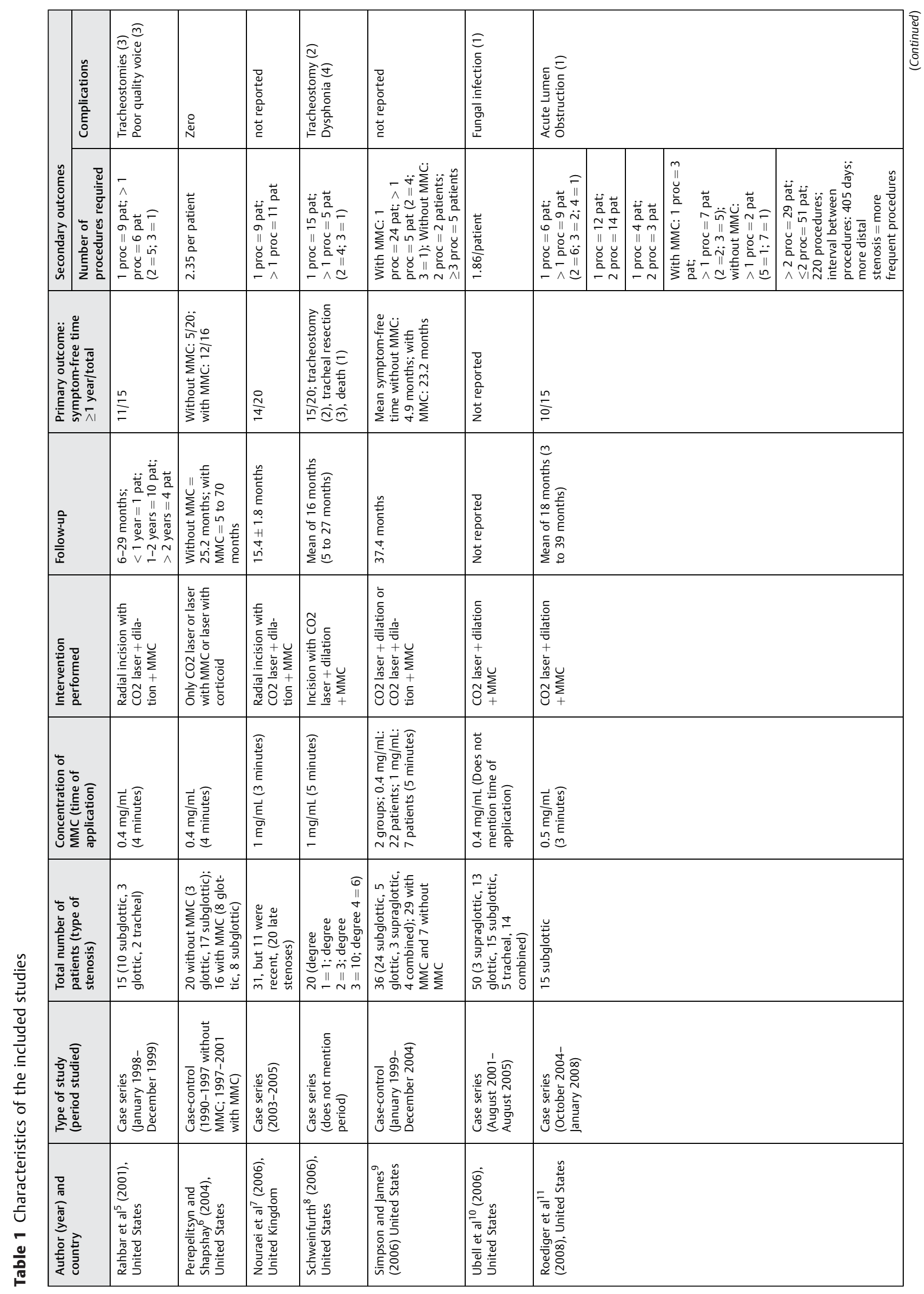




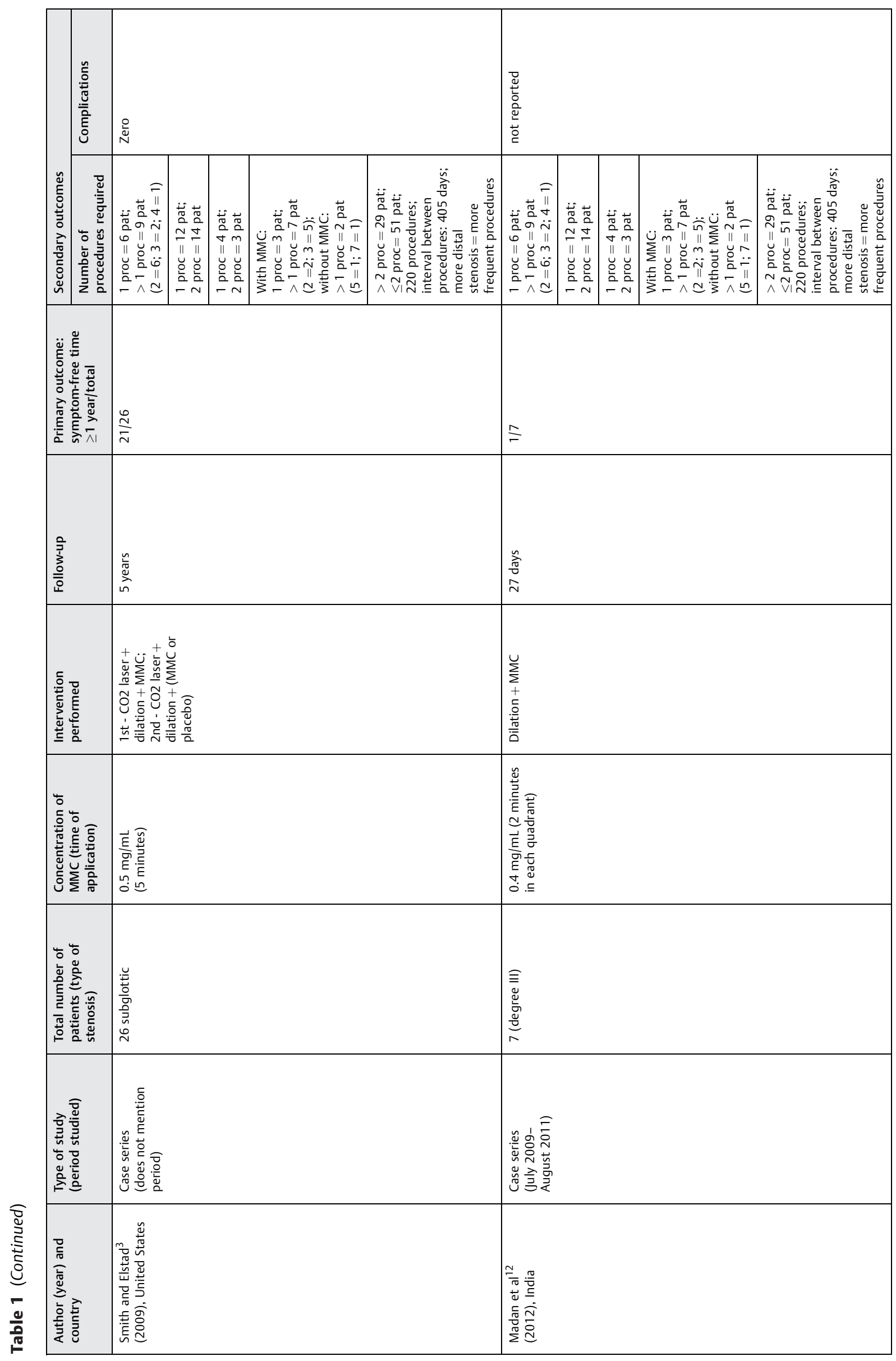




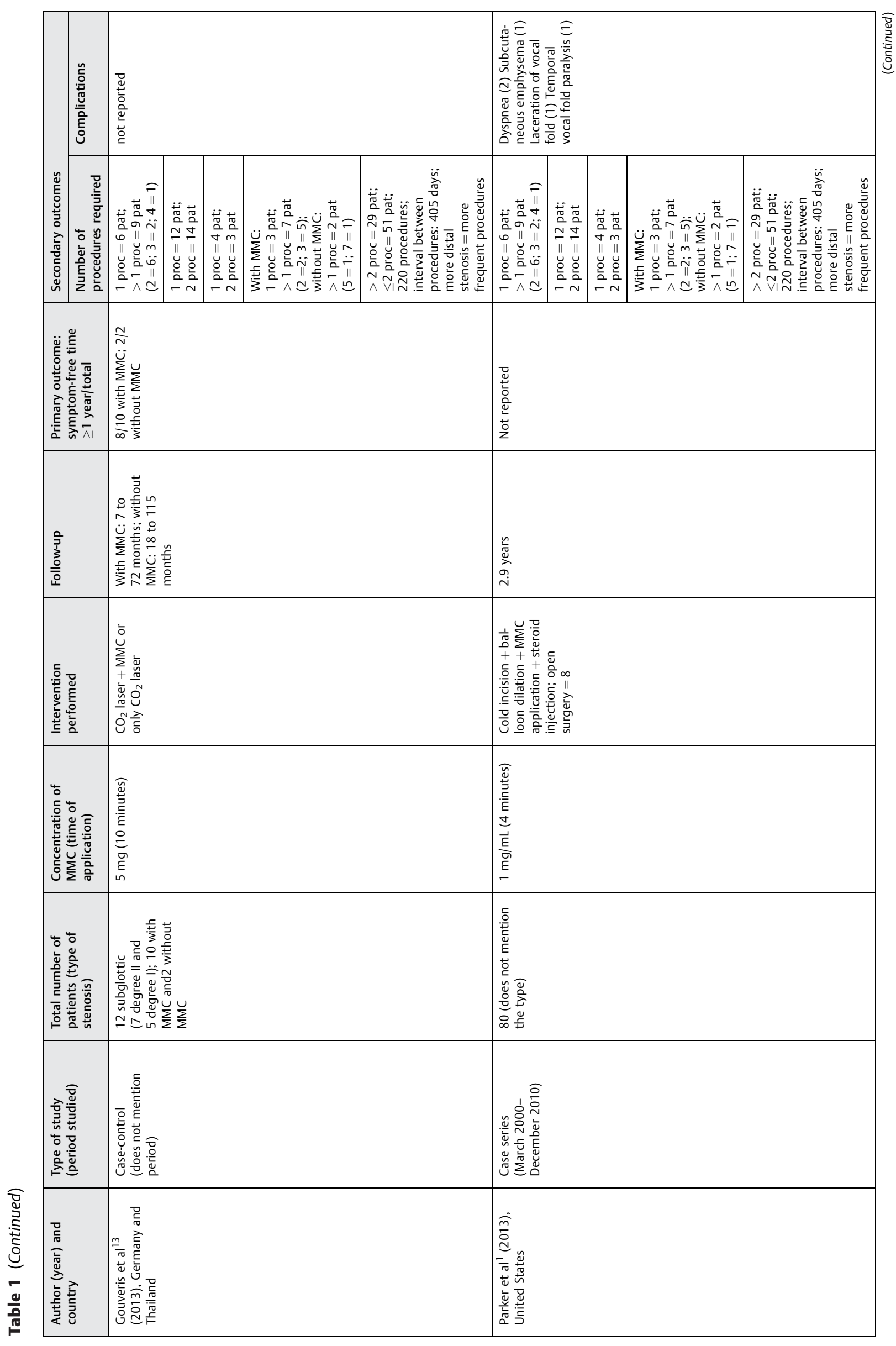




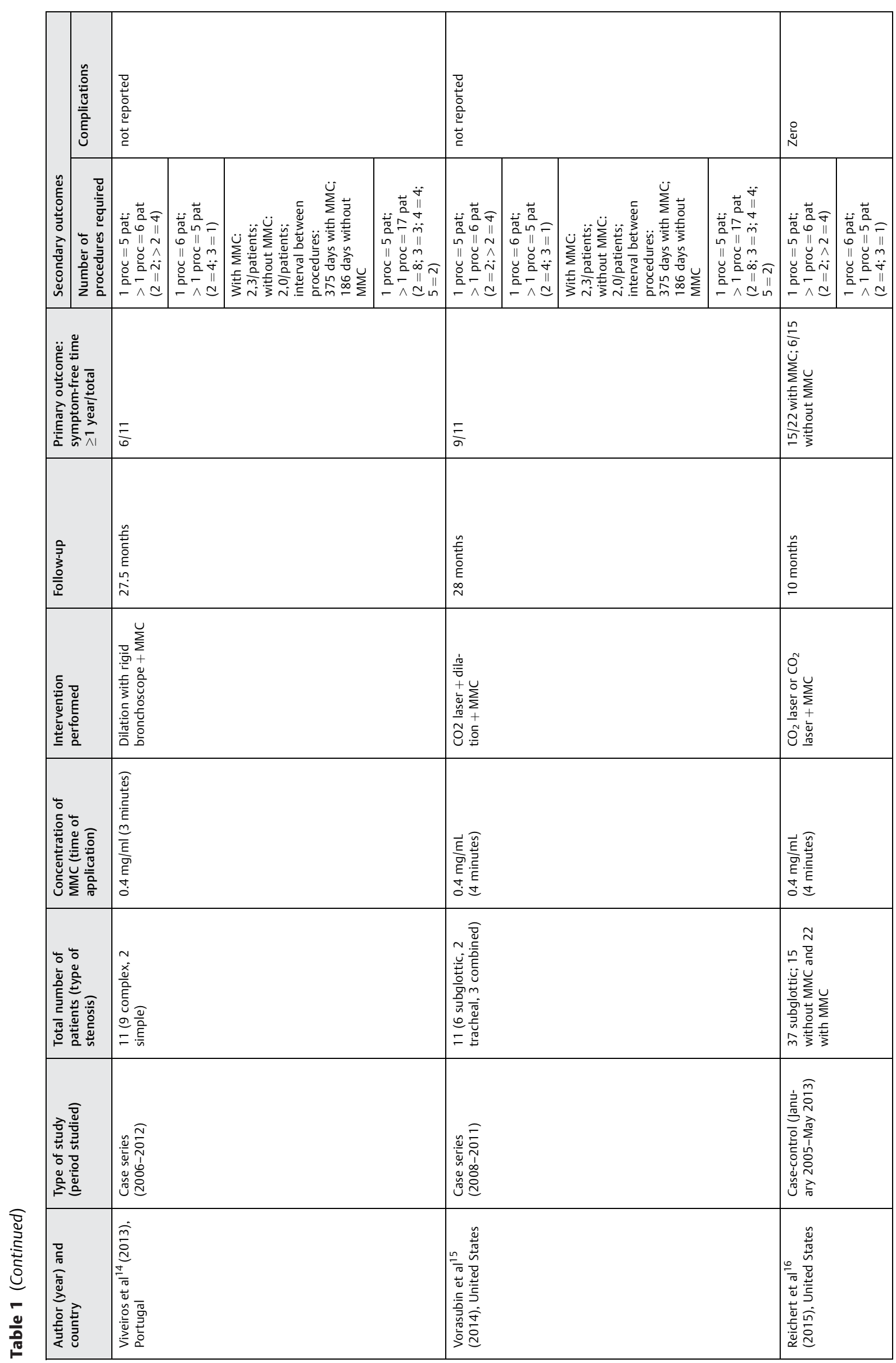




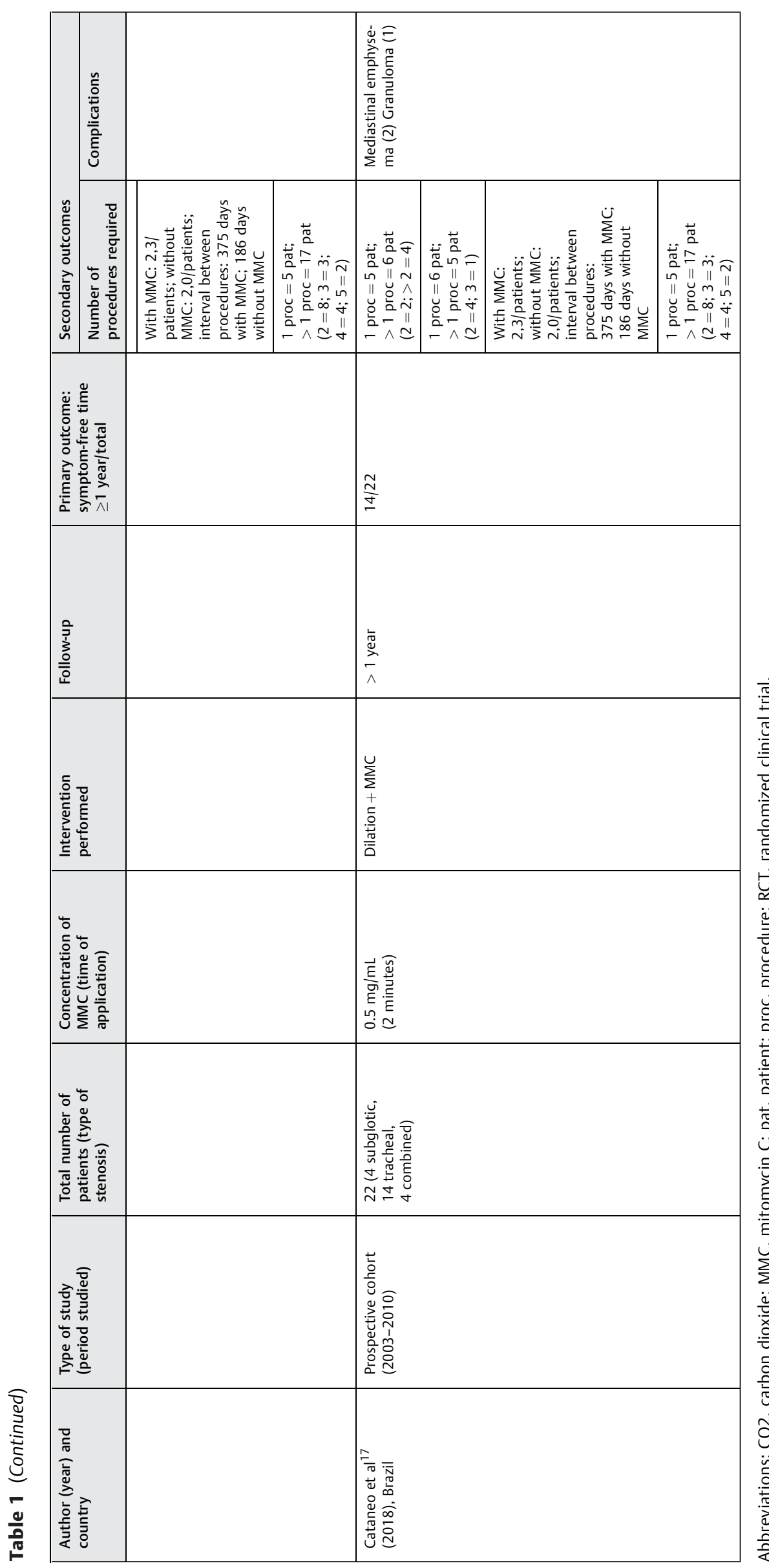

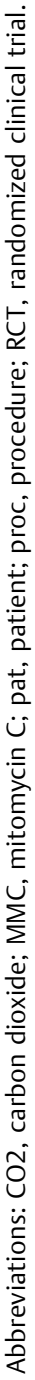


of stenosis in each study differed, and not all studies classified the degree of stenosis at the time of diagnosis before the interventions. Of the 387 patients, 29 had diagnoses of glottic stenosis, 135 of subglottic stenosis, 7 of supraglottic stenosis, 23 of tracheal stenosis, and 86 of combined-site stenosis. The remaining 107 patients were distributed among 6 studies in which the location of the stenosis was not mentioned. Of the 387 patients, the degree of stenosis was only determined in 64 patients. It was not possible to perform subgroup analyses because the outcomes were not separated by location and severity of the stenosis. Two different interventions, with and without MMC, were performed in four studies. ${ }^{6,9,13,16}$ In the remaining studies, the intervention was always with MMC. The type of intervention in each study, the dose and time of MMC application, the number of procedures performed, the follow-up period, and the treatment outcomes are detailed in -Table 1.

Risk of bias: only one study was an RCT in which MMC was used in all patients in the first procedure, and all of them were randomized to receive either MMC or placebo in the second procedure. $^{3}$ This randomization was performed to test whether two applications of MMC were better than one application. As MMC was administered to all patients once or twice, they were used in the meta-analysis, so despite the fact that it was an RCT study, the data were used as a series of cases. The other studies had an observational design and, therefore, were subject to the effect of confounding factors. A prospective, double-blinded, randomized, placebo-controlled ongoing study was identified in the Clinical Trials database(NCT01523275). The University of California, San Francisco, has been recruiting participants since January 2012, the study is scheduled to be completed by January 2019, and no preliminary data had been published until November 27, 2019. This study includes individuals older than 18 years with laryngotracheal stenosis, and it aims to determine if the application of MMC associated with radial incision using $\mathrm{CO}_{2}$ laser and dilation increases the interval between the endoscopic surgical procedures, improves the symptom-free period, and determines the maximum inspiratory flow in patients with laryngotracheal stenosis at a followup period of 24 months. ${ }^{18}$

\section{Effects of the Interventions (Meta-Analysis)}

Full or partial resolution: of the 15 studies selected, 12 analyzed the symptom-free period after the use of MMC $(n=195)$. The rate of symptom-free periods $\geq 1$ year was of $69 \%$ with the use of MMC (95\%CI: $61-76 \%, \mathrm{I}^{2}=17.3 \%$ ) (-Fig. 2).

Four studies analyzed the symptom-free period with and without the use of MMC. However, only 3 of these studies ( $n=85,48$ with and 37 without MMC) reported the number of patients who remained symptom free for $\geq 1$ year, which was 73\% for MMC users and 35\% for non-MMC users. The other study presented the mean symptom-free period of 23.2 months for those who received MMC, and of 4.9 months for those who did not receive MMC. The meta-analysis comparing these two groups showed that the odds of being

\section{Proportion meta-analysis plot [random effects]}

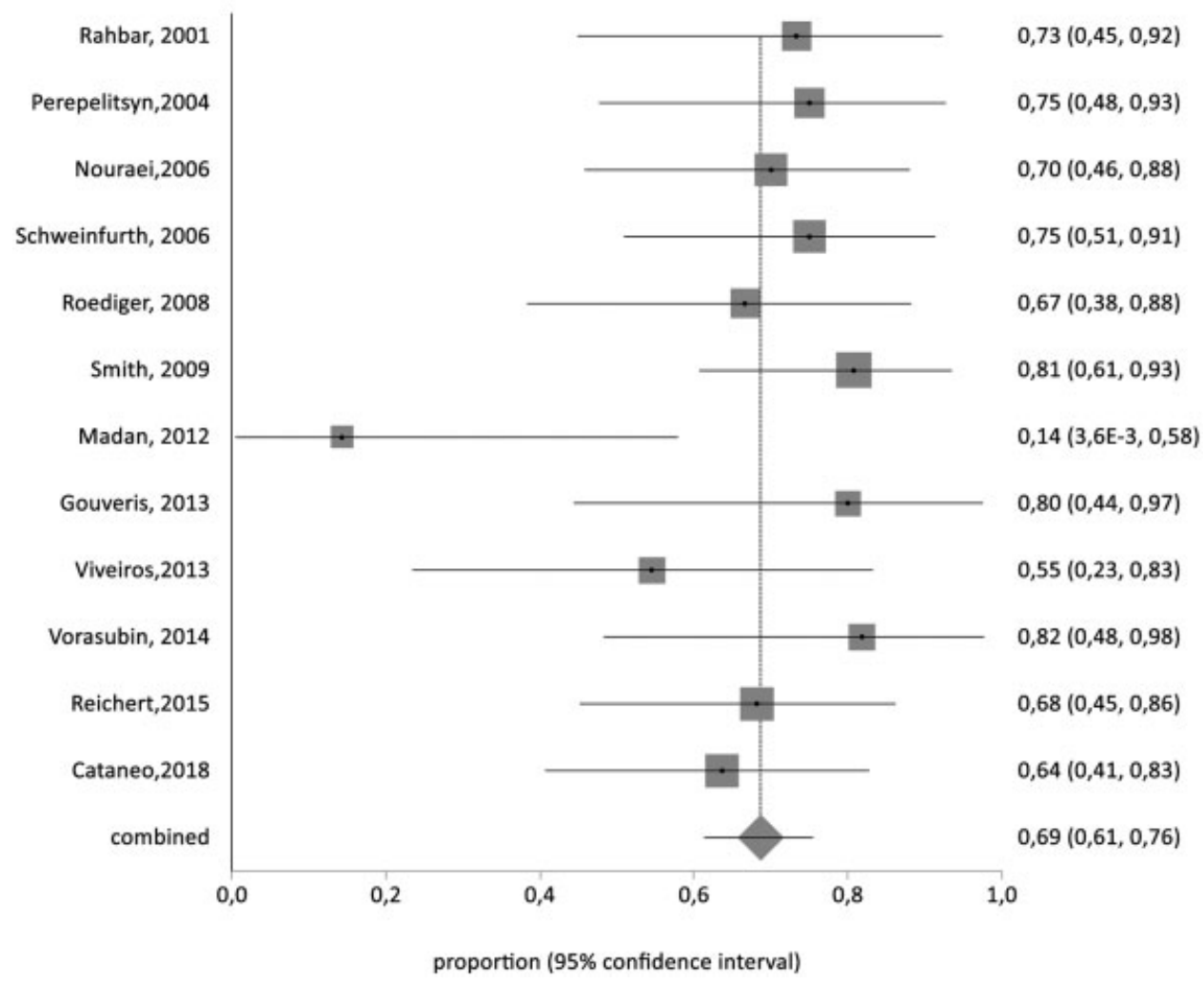

Fig. 2 Forest plot of symptom-free period $\geq 1$ year for patients receiving MMC. The proportion of patients with this outcome was of 0.69 (95\%Cl: $0.61-0.76$ ). 


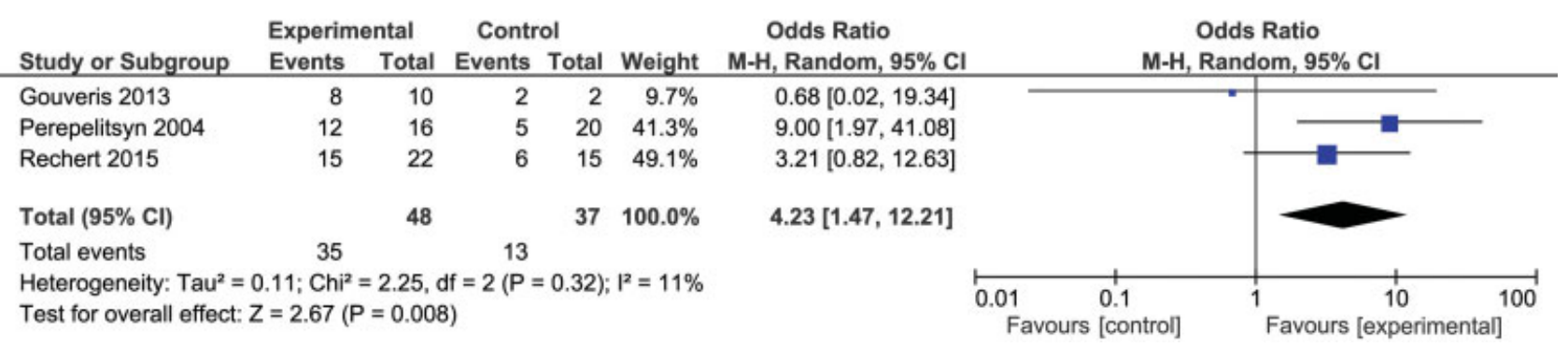

Fig. 3 Forest plot of symptom-free period $\geq 1$ year. The chance for this outcome was greater in patients who received $\mathrm{MMC}(\mathrm{OR}: 4.23 ; 95 \% \mathrm{Cl}$ : $\left.1.47-12.21 ;\left.\right|^{2}=11 \%\right)$.

symptom-free for $\geq 1$ year was 4 times higher in MMC-treated patients (OR: 4.23; 95\%CI: 1.47-12.21; $\mathrm{I}^{2}=11 \%$ ) (-Fig. 3).

Number of procedures required: in 11 of the studies, it was possible to separate the patients into 2 groups, those submitted to a single procedure and those who underwent more than one procedure. A single procedure was performed in 52\% of the patients (95\%CI: $39-64 \%$; $\mathrm{I}^{2}=64.7 \%$ ) (- Fig. 4A) and more than 1 procedure was performed in $48 \%$ of the patients (95\%CI: 36-61\%; $\mathrm{I}^{2}=64.7 \%$ ) (-Fig. 4B).

Complications: The complications were considered to be related to the entire endoscopic procedure, and not only to the application of MMC. Of the studies analyzed, 6 reported complications, and 3 reported no complications (complications: 9\%; 95\%CI: 3-18\%; $\mathrm{I}^{2}=79.8 \%$; studies: 9; participants: 285) (-Fig. 5). Six studies did not report this outcome. The complications included tracheostomy, dysphonia, mediastinal and subcutaneous emphysema, fungal infection at the stenotic site, acute light obstruction, restenosis, vocal fold laceration, granuloma, and temporary vocal paralysis.

Other outcomes assessed: one study evaluated the total cost involved in treating patients with MMC. ${ }^{10}$ Endoscopic treatment using MMC was reported to cost US\$455 per patient, and open surgery, US\$7,840 (17 times more expensive). The study authors concluded that the endoscopic treatment is economical if only 1 in 17 patients does not require open surgery. One study measured airway resistance (AR) before and after the procedure. ${ }^{13}$ The average AR was reduced by almost half, from $1.004 \mathrm{kPa} / \mathrm{L} / \mathrm{s}$ before the procedure to $0.526 \mathrm{kPa} / \mathrm{L} / \mathrm{s}$ after the procedure.

\section{Discussion}

The surgical treatment of laryngotracheal stenosis by resection of the stenotic segment followed by end-to-end anastomosis has been shown to be highly effective; however, not all patients are indicated for this surgery. The endoscopic treatment that offers dilation of the stenotic area is an alternative option for such patients, although scarring of the bloody area during the treatment leads to recurrence of the stenosis. Drugs that prevent the proliferation of fibroblasts, such as MMC, are reported to delay restenosis. Mitomycin C was first used for airway dilation in the 1970s, and since then, the results of the studies have been ambiguous, with some proving its efficacy ${ }^{19,20}$ and others attesting its ineffectiveness. $^{12}$

Despite the fact that the prevalence of laryngotracheal stenosis is on the rise, the incidence is relatively low. This rise can be attributed to treatment success in intensive care units, which are currently saving several patients who in the past would have relatively low survival rates. Perhaps because of the low incidence, there are no published randomized studies comparing the use of MMC with placebo or other drugs, and healthcare providers have to rely on observational studies for decision making, and even carry out systematic reviews with them, until randomized studies are performed. ${ }^{21}$
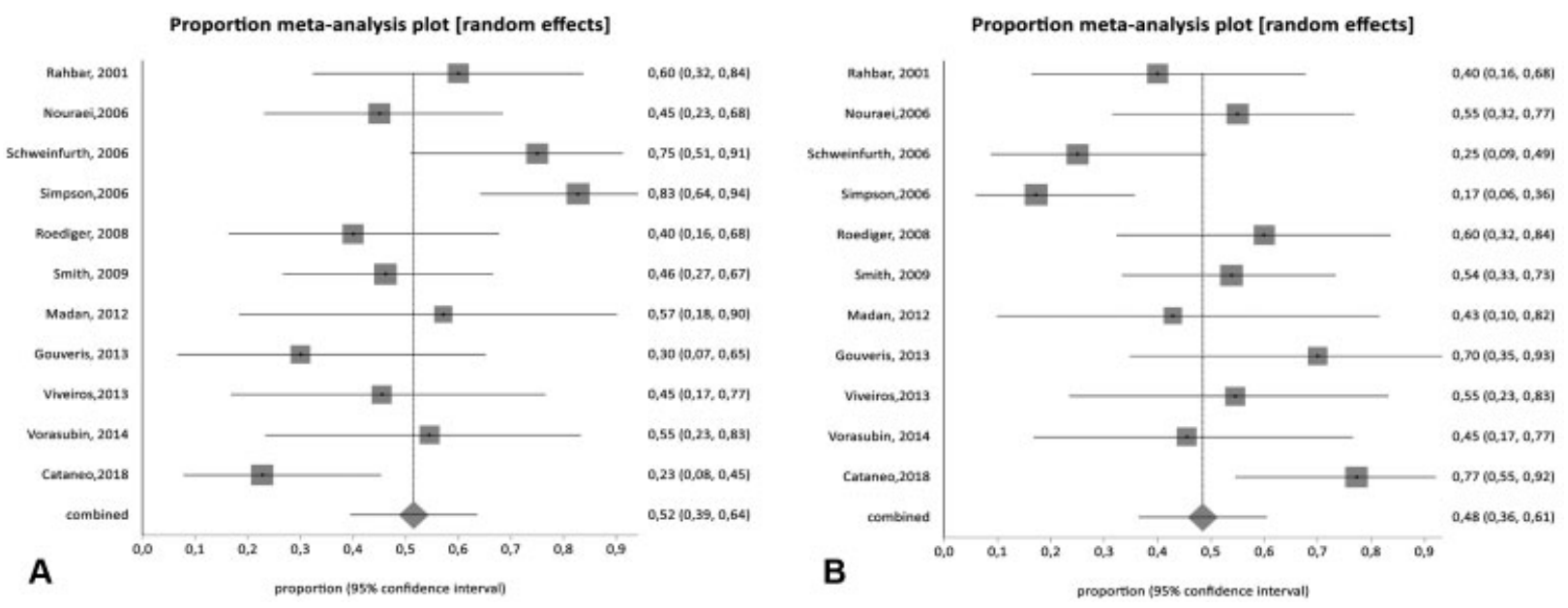

Fig. 4 (A) Proportion of patients who underwent a single procedure (0.52; 95\% Cl: 0.39-0.64). (B) Proportion of patients who underwent more than one procedure $(0.48 ; 95 \% \mathrm{Cl}: 0.36-0.61)$. 


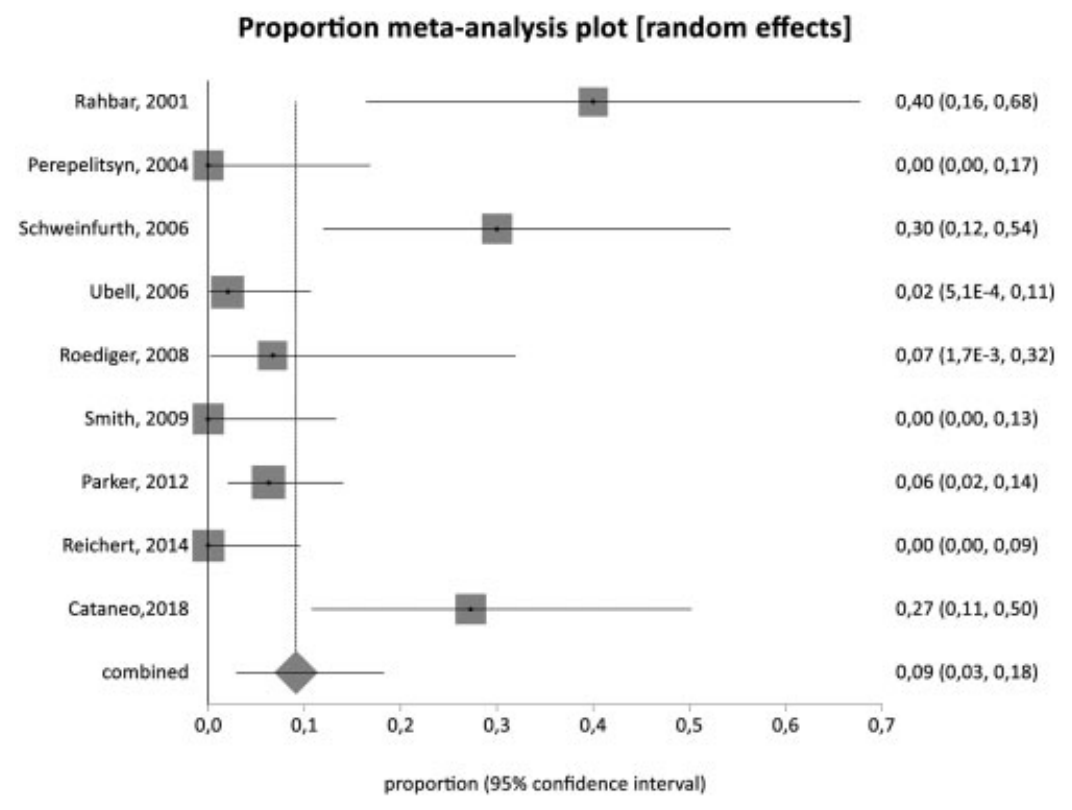

Fig. 5 Proportion of complications in 9 studies $(0.09 ; 95 \% \mathrm{Cl}$ : $0.03-0.18)$.

The present review indicates that MMC is effective in the treatment of laryngotracheal stenosis, with a resolution probability characterized by symptom-free period $\geq 1$ year of $\sim 70 \%$. This result is promising and optimistic for the adjuvant use of topical MMC in the conservative treatment of laryngotracheal stenosis of varying degrees and durations, as the studies evaluated patients with various types of stenosis with different characteristics. Most of the studies in the present review showed that the benefits outweighed the risks associated with the use of MMC, and that the symptomfree period was $\geq 1$ year in most patients.

The meta-analysis of the studies comparing endoscopic treatments with and without MMC showed that the chance of achieving a symptom-free period $\geq 1$ year is 4 times higher in patients receiving MMC. However, the number of studies evaluating patients who did not receive MMC was considerably low, with a small sample size, resulting in a large dilation of the CI. Therefore, the evidence will have to come from future studies.

Regarding the number of procedures required, we found no difference between the patients who underwent a single procedure and those who were submitted to more than one procedure. Moreover, we found only 1 randomized, prospective, double-blinded, placebo-controlled study with 26 patients with laryngotracheal stenosis. In the first procedure, all patients underwent a radial laser incision using $\mathrm{CO}_{2}$ laser, dilation, and topical application of MMC $(0.5 \mathrm{mg} / \mathrm{mL})$. In the second procedure, after 1 month, these patients were randomized to receive either MMC or a placebo. During the first 3 years of follow-up, the study showed that patients who received MMC in both procedures had a higher rate of resolution of symptoms than those who received MMC only in the first procedure. Nevertheless, after the fourth year, the frequency of restenosis was almost the same between the two groups.

Complications were only reported in 9 studies, but with quite different incidences: 6 of them with low and 3 with a high frequency of complications, so the heterogeneity among studies exceeded $75 \%$, which makes the combination of studies inappropriate, so the incidence of complications should be viewed with caution. These complications were not specific to the procedure; they were also related to the disease, such as fungal infection and the need for tracheostomy. The complications resulting from the intervention were also not specific because of the use of MMC, but of the entire endoscopic procedure, such as emphysema, dysphonia, laceration, or vocal fold paralysis and acute light obstruction, which occurred with a low incidence. This incidence was calculated based on the number of patients, and it was lower regarding the number of procedures, because many of the patients underwent more than one procedure.

Most of the included studies were considered to be affected by confounding factors because of their observational design, thereby reducing the quality of the evidence. The only randomized study considered to have low risk of bias compared one with two applications of MMC, not MMC with placebo; therefore, it cannot be used to increase the quality of evidence in the present review.

At the time of the database search, we found a prospective, randomized, double-blinded, placebo-controlled study that is still in the recruitment phase, which plans to use topical MMC as adjuvant therapy to control stenosis. Whether this study presents a relevant method and more reliable results following the application of MMC will only be known after its publication. ${ }^{18}$

We are confident that well-focused clinical issues have facilitated the construction of a comprehensive search strategy suitable to capture most of the relevant literature published, minimizing the likelihood of missing relevant studies. We believe that the well-defined criteria for the inclusion of the studies and the standardized and unbiased extraction of data internally validated the review, although most of the studies were observational. 
The inclusion of observational studies in systematic reviews has already been done in a Cochrane review. ${ }^{22}$ Some characteristics of observational studies may reduce the risk of bias. For instance, a low selection bias is introduced if the control group is selected from the same population of cases; the performance bias is often high when the participants and the professionals are not masked; and the detection bias may be low if the results are obtained from medical records, resulting in the outcome assessors being independent. ${ }^{21}$

The broad search strategy applied to the various databases may have reduced the loss of primary studies that used MMC in the conservative treatment of laryngotracheal stenosis.

Despite the poor quality of the evidence, the present meta-analysis can be consulted for decision-making on the use of topical MMC in the treatment laryngotracheal stenosis as an alternative option. It also suggests the need for prospective studies comparing MMC with placebo or other drugs to clarify the efficacy of the MMC therapy.

\section{Final Comments}

Evidence supports the effective and safe use of topical MMC as an adjuvant in the endoscopic treatment of laryngotracheal stenosis.

Implications for the practice: topical MMC seems to provide a reasonable effectiveness, with a symptom-free period $\geq 1$ year. The safety in the performance of the procedure is difficult to measure by the incidence of complications. This is due to the heterogeneity among the studies, some with a high and others with a low incidence of complications.

Implications for research: there is a greater need for highquality prospective, controlled trials comparing the topical use of MMC with other types of conservative treatment for laryngotracheal stenosis to provide an alternative treatment option for patients not indicated for open surgery.

Conflict of Interests Statement and Sources of Funding The authors have none to disclose.

\section{References}

1 Parker NP, Bandyopadhyay D, Misono S, Goding GS Jr. Endoscopic cold incision, balloon dilation, mitomycin $\mathrm{C}$ application, and steroid injection for adult laryngotracheal stenosis. Laryngoscope 2013;123(01):220-225. Doi: 10.1002/lary.23638

2 Garrett CG, Soto J, Riddick J, Billante CR, Reinisch L. Effect of mitomycin-C on vocal fold healing in a canine model. Ann Otol Rhinol Laryngol 2001;110(01):25-30

3 Smith ME, Elstad M. Mitomycin C and the endoscopic treatment of laryngotracheal stenosis: are two applications better than one? Laryngoscope 2009;119(02):272-283. Doi: 10.1002/lary.20056

4 El Dib R, Nascimento Junior P, Kapoor A. An alternative approach to deal with the absence of clinical trials: a proportional metaanalysis of case series studies. Acta Cir Bras 2013;28(12): 870-876. Doi: 10.1590/S0102-86502013001200010

5 Rahbar R, Shapshay SM, Healy GB. Mitomycin: effects on laryngeal and tracheal stenosis, benefits, and complications. Ann Otol Rhinol Laryngol 2001;110(01):1-6. Doi: 10.1177/000348940111000101
6 Perepelitsyn I, Shapshay SM. Endoscopic treatment of laryngeal and tracheal stenosis-has mitomycin $\mathrm{C}$ improved the outcome? Otolaryngol Head Neck Surg 2004;131(01):16-20. Doi: 10.1016/j. otohns.2004.03.001

7 Nouraei SA, Singh A, Patel A, Ferguson C, Howard DJ, Sandhu GS. Early endoscopic treatment of acute inflammatory airway lesions improves the outcome of postintubation airway stenosis. Laryngoscope 2006;116(08):1417-1421. Doi: 10.1177/000348940611 500105

8 Schweinfurth JM. Endoscopic treatment of severe tracheal stenosis. Ann Otol Rhinol Laryngol 2006;115(01):30-34. Doi: 10.1177/00034 8940611500105

9 Simpson CB, James JC. The efficacy of mitomycin-C in the treatment of laryngotracheal stenosis. Laryngoscope 2006;116(10): 1923-1925. Doi: 10.1097/01.mlg.0000235934.27964.88

10 Ubell ML, Ettema SL, Toohill RJ, Simpson CB, Merati AL. Mitomycin-c application in airway stenosis surgery: analysis of safety and costs. Otolaryngol Head Neck Surg 2006;134(03):403-406. Doi: $10.1016 /$ j.otohns.2005.10.057

11 Roediger FC, Orloff LA, Courey MS. Adult subglottic stenosis: management with laser incisions and mitomycin-C. Laryngoscope 2008;118(09):1542-1546. Doi: 10.1097/MLG.0b013e318179247a

12 Madan K, Agarwal R, Aggarwal AN, Gupta D. Utility of rigid bronchoscopic dilatation and mitomycin $C$ application in the management of postintubation tracheal stenosis: case series and systematic review of literature. J Bronchology Interv Pulmonol 2012;19(04):304-310. Doi: 10.1097/LBR.0b013e3182721290

13 Gouveris H, Karaiskaki N, Koutsimpelas D, Chongolwatana C, Mann W. Treatment for adult idiopathic and Wegener-associated subglottic stenosis. Eur Arch Otorhinolaryngol 2013;270(03): 989-993. Doi: 10.1007/s00405-012-2240-z

14 Viveiros F, Gomes J, Oliveira A, Neves S, Almeida J, Moura e Sá J. Aplicação tópica de mitomicina-C como adjuvante no tratamento broncoscópico da estenose traqueal pós-entubação. Rev Port Pneumol 2013;19(06):276-280. Doi: 10.1016/j.rppneu.2013.06.006

15 Vorasubin N, Vira D, Jamal N, Chhetri DK. Airway management and endoscopic treatment of subglottic and tracheal stenosis: the laryngeal mask airway technique. Ann Otol Rhinol Laryngol 2014; 123(04):293-298. Doi: 10.1177/0003489414525340

16 Reichert LK, Zhao AS, Galati LT, Shapshay SM. The efficacy of mitomycin $c$ in the treatment of laringotracheal stenosis: results and experiences with a difficult disease entity. ORL J Otorhinolaryngol Relat Spec 2015;77(06):351-358. Doi: 10.1159/000439174

17 Cataneo DC, Ximenes AMG, Cataneo AJM. Mitomycin C in the endoscopic treatment of tracheal stenosis: a prospective cohort study. J Bras Pneumol 2018;44(06):486-490. Doi: 10.1590/S180637562017000000423

18 Yung KC, Chang J, Courey MS A. A randomized controlled trial of adjuvant mitomycin-c in endoscopic surgery for laryngotracheal stenosis. Laryngoscope. 2019 Apr 25. doi: 10.1002/lary.28025. [Epub ahead of print]

19 Correa AJ, Reinisch L, Sanders DL, et al. Inhibition of subglottic stenosis with mitomycin-C in the canine model. Ann Otol Rhinol Laryngol 1999;108(11 Pt 1):1053-1060

20 Spector JE, Werkhaven JA, Spector NC, et al. Preservation of function and histologic appearance in the injured glottis with topical mitomycin-C. Laryngoscope 1999;109(7 Pt 1):1125-1129. Doi: 10.1097/00005537-199907000-00022

21 O'Neil M, Berkman N, Hartling L, et al. Observational evidence and strength of evidence domains: case examples. Syst Rev 2014;3:35. Doi: 10.1186/2046-4053-3-35

22 Thompson DC, Rivara FP, Thompson R. Helmets for preventing head and facial injuries in bicyclists. Cochrane Database Syst Rev 2000;(02):CD001855. Doi: 10.1002/14651858.CD001855 